\title{
Blind Recognition of Linear Space-Time Block Codes: A Likelihood-Based Approach
}

\author{
Vincent Choqueuse, Member, IEEE, Mélanie Marazin, Ludovic Collin, Koffi Clément Yao, and \\ Gilles Burel, Senior Member, IEEE
}

\begin{abstract}
Blind recognition of communication parameters is a research topic of high importance for both military and civilian communication systems. Numerous studies about carrier frequency estimation, modulation recognition as well as channel identification are available in literature. This paper deals with the blind recognition of the space-time block coding (STBC) scheme used in multiple-input-multiple-output (MIMO) communication systems. Assuming there is perfect synchronization at the receiver side, this paper proposes three maximum-likelihood (ML)-based approaches for STBC classification: the optimal classifier, the second-order statistic (SOS) classifier, and the code parameter (CP) classifier. While the optimal and the SOS approaches require ideal conditions, the $\mathrm{CP}$ classifier is well suited for the blind context where the communication parameters are unknown at the receiver side. Our simulations show that this blind classifier is more easily implemented and yields better performance than those available in literature.
\end{abstract}

Index Terms-Maximum-likelihood (ML) detection, multiple-input-multiple-output (MIMO) systems, space-time block code (STBC).

\section{INTRODUCTION}

B LIND recognition of communication parameters is an intermediate step between signal detection and signal decoding/demodulation. In civilian applications, blind recognition algorithms are used in software-defined radio (SDR) to cope with a large panel of communication systems. In electronic warfare, these algorithms are required for signal interception and processing, two tasks of key importance in tactical operations. Usually, the largest part of the algorithms is devoted to the blind recognition of single-input-single-output (SISO) communication parameters. Other investigations have dealt with the development of new technologies aimed at enhancing the reliability of data transmission in wireless communication systems. Among them, one of the most promising technologies is based on the use of multiple-input-multiple-output (MIMO)

Manuscript received February 16, 2009; accepted September 30, 2009. First published November 06, 2009; current version published February 10, 2010. The associate editor coordinating the review of this manuscript and approving it for publication was Prof. Roberto Lopez-Valcarce.

V. Choqueuse was with Lab-STICC, Université de Brest, 29238 Brest Cedex 3, France. He is now with LBMS, UMR CNRS 3192, ISSTB, 29238 Brest Cedex

3, France (e-mail: vincent.choqueuse@gmail.com).

M. Marazin, L. Collin, K. Yao, and G. Burel are with the Laboratory for Sciences and Technologies of Information, Communication and Knowledge (LabSTICC-UMR CNRS 3192), Université de Brest, 29238 Brest Cedex 3, France (e-mail: melanie.marazin@univ-brest.fr; Ludovic.Collin@univ-brest.fr; KoffiClement.Yao@univ-brest.fr; Gilles.Burel@univ-brest.fr).

Color versions of one or more of the figures in this paper are available online at http://ieeexplore.ieee.org.

Digital Object Identifier 10.1109/TSP.2009.2036062 systems [1] in conjunction with space-time block coding (STBC) [2]-[4]. The principle of STBC is to take advantage of channel diversity through a proper encoding of the data streams into structured blocks. MIMO-STBC communication systems have been recently standardized in IEEE 802.16e and IEEE $802.11 \mathrm{n}$ and appear as an ideal technology for the next generation of wireless products.

The development of blind receivers coping with MIMO communication systems is a new challenge. The blind decoding of the received symbols requires the estimation of several parameters like the number of transmitter antennas, the space-time coding, the modulation, and the channel. Some investigations have been focused on the blind estimation of either the number of transmitters [5], [6] or the modulation [7]. Furthermore, other methods devoted to the channel estimation in space-time coding systems are also available in [8]-[14]. However, these algorithms require the knowledge of the space-time coding at the receiver side. The blind STBC recognition is an emerging topic, which has been recently addressed in [15]-[18]. These featurebased methods exploit the space-time redundancy of the received samples to discriminate between several STBCs. The redundancy is measured through space-time correlations and the automatic classification is performed through hypothesis testing [15]-[17] or by minimizing a cost function [18]. In the study reported here, the maximum-likelihood (ML) approach is applied for STBC recognition. The ML approach is a powerful classification tool, which has been previously employed for modulation-recognition problems [19]-[21].

The paper is organized as follows. Section II introduces the signal models and the assumptions made in the study. Section III describes the optimal classifier in the Bayesian sense. Section IV presents a low-complexity second-order statistic classifier (SOS) based on the relaxation of the finite alphabet property of sources. Finally, Section V describes a simple code parameter (CP) classifier, which can be employed to discriminate between several STBCs with different code rate or code length. Finally, the performances of the three methods are reported in Section VI.

\section{Signal Models AND Assumptions}

\section{A. Signal Model of Linear STBC}

Let us consider a linear $\operatorname{STBC}\left(n_{t}, n, l\right)$ that transmits $n$ symbols during $l$ time slots and uses $n_{t}$ antennas at the transmitter side. The space-time block encoder generates an $n_{t} \times l$ block matrix, denoted by $\mathbf{C}(\mathbf{s})$, from a block of $n$ symbols denoted $\mathbf{s}=\left[s_{1}, \cdots, s_{n}\right]^{T}$. The general expression of $\mathbf{C}(\mathbf{s})$ is

$$
\mathbf{C}(\mathbf{s})=\left[\mathbf{A}_{1} \tilde{\mathbf{s}}, \cdots, \mathbf{A}_{l} \tilde{\mathbf{s}}\right]
$$


where the $2 n$ vector $\tilde{\mathbf{s}}=\left[\Re e\left(\mathbf{s}^{T}\right), \Im m\left(\mathbf{s}^{T}\right)\right]^{T}$ corresponds to the vertical concatenation of the real and imaginary parts of $\mathbf{s}$. The $n_{t} \times 2 n$ matrices $\mathbf{A}_{u}(1 \leq u \leq l)$ correspond to the coding matrices and depend only on the STBC employed at the transmitter side. The class of STBC is large and contains, for example, the spatial multiplexing, the linear dispersion codes [22], the quasi-orthogonal codes (QOSTBC) [23], and the orthogonal codes (OSTBC) [24], [25].

1) Example-Spatial Multiplexing: The $\operatorname{STBC}\left(n_{t}, n_{t}, 1\right)$ called spatial multiplexing ${ }^{1}$ encodes a block of $n_{t}$ symbols into an $n_{t} \times 1$ matrix $\mathbf{C}(\mathbf{s})$ as

$$
\mathbf{C}(\mathbf{s})=\left[\begin{array}{c}
s_{1} \\
\vdots \\
s_{n}
\end{array}\right] .
$$

The coding matrix $\mathbf{A}_{1}$ is given by

$$
\mathbf{A}_{1}=\left[\mathbf{I}_{n_{t}} j \mathbf{I}_{n_{t}}\right]
$$

where $\mathbf{I}_{n_{t}}$ is the identity matrix of size $n_{t} \times n_{t}$.

2) Example-Alamouti Code: The $\operatorname{STBC}(2,2,2)$ called Alamouti code [24] encodes a block of two symbols into a $2 \times 2$ matrix $\mathbf{C}(\mathbf{s})$ as

$$
\mathbf{C}(\mathbf{s})=\left[\begin{array}{cc}
s_{1} & -s_{2}^{*} \\
s_{2} & s_{1}^{*}
\end{array}\right] \text {. }
$$

The two coding matrices $\mathbf{A}_{1}$ and $\mathbf{A}_{2}$ are written as

$$
\begin{aligned}
\mathbf{A}_{1} & =\left[\begin{array}{llll}
1 & 0 & j & 0 \\
0 & 1 & 0 & j
\end{array}\right] \\
\mathbf{A}_{2} & =\left[\begin{array}{cccc}
0 & -1 & 0 & j \\
1 & 0 & -j & 0
\end{array}\right] .
\end{aligned}
$$

\section{B. Signal Model of the Communication}

In this paper, the receiver is assumed to be perfectly synchronized with the transmitter, i.e., one sample per symbol and an optimum sampling time. In a noncooperative context, the synchronization can be achieved through a symbol-timing estimator based on the squaring algorithm [26] or by exploiting the cyclic correlations of the received samples [27].

Let us assume a quasi-static frequency-flat MIMO channel modeled by an $n_{r} \times n_{t}$ matrix $\mathbf{H}$ where $n_{r}$ corresponds to the number of receiver antennas. By assuming a perfect synchronization, the $v$ th received block $\mathbf{Y}_{v}$ is expressed as

$$
\mathbf{Y}_{v}=\mathbf{H C}(\mathbf{s})+\mathbf{B}_{v}
$$

where the $n_{r} \times l$ matrix $\mathbf{B}_{v}=\left[\mathbf{b}_{v}(0), \cdots, \mathbf{b}_{v}(l-1)\right]$ refers to the additive noise. By stacking the real and imaginary parts of the data, one obtains

$$
\left[\begin{array}{l}
\Re e\left(\mathbf{Y}_{v}\right) \\
\Im m\left(\mathbf{Y}_{v}\right)
\end{array}\right]=\overline{\mathbf{H}}\left[\begin{array}{l}
\Re e(\mathbf{C}(\mathbf{s})) \\
\Im m(\mathbf{C}(\mathbf{s}))
\end{array}\right]+\left[\begin{array}{l}
\Re e\left(\mathbf{B}_{v}\right) \\
\Im m\left(\mathbf{B}_{v}\right)
\end{array}\right]
$$

where the $2 n_{r} \times 2 n_{t}$ matrix $\overline{\mathbf{H}}$ is given by

$$
\overline{\mathbf{H}}=\left[\begin{array}{cc}
\Re e(\mathbf{H}) & -\Im m(\mathbf{H}) \\
\Im m(\mathbf{H}) & \Re e(\mathbf{H})
\end{array}\right] .
$$

\footnotetext{
${ }^{1}$ In the strict sense, the spatial multiplexing is not a space-time code since it
} does not achieve space-time diversity.
In this paper, the received blocks are represented as column vectors. Let us denote by $\tilde{\mathbf{y}}_{v}$ and $\tilde{\mathbf{b}}_{v}$ the column vectors of size $2 n_{r} l$, which are defined as

$$
\tilde{\mathbf{y}}_{v}=\operatorname{vec}\left\{\begin{array}{c}
\Re e\left(\mathbf{Y}_{v}\right) \\
\Im m\left(\mathbf{Y}_{v}\right)
\end{array}\right\} \quad \tilde{\mathbf{b}}_{v}=\operatorname{vec}\left\{\begin{array}{c}
\Re e\left(\mathbf{B}_{v}\right) \\
\Im m\left(\mathbf{B}_{v}\right)
\end{array}\right\}
$$

where vec $\{$.$\} represents vectorization. Under these notations,$ (8) can be expressed as (see [28])

$$
\tilde{\mathbf{y}}_{v}=\left(\mathbf{I}_{l} \otimes \overline{\mathbf{H}}\right) \operatorname{vec}\left\{\begin{array}{c}
\Re e(\mathbf{C}(\mathbf{s})) \\
\Im m(\mathbf{C}(\mathbf{s}))
\end{array}\right\}+\tilde{\mathbf{b}}_{v}
$$

where $\otimes$ corresponds to the Kronecker product. Finally, the use of (11) and (1) leads to

$$
\tilde{\mathbf{y}}_{v}=\left(\mathbf{I}_{l} \otimes \overline{\mathbf{H}}\right) \mathbf{M}_{\mathcal{C}} \tilde{\mathbf{s}}_{v}+\tilde{\mathbf{b}}_{v}
$$

where the $2 l_{n} \times 2 n$ matrix $\mathbf{M}_{\mathcal{C}}$ depends only on the STBC $\mathcal{C}$ and is defined as

$$
\mathbf{M}_{\mathcal{C}}=\left[\begin{array}{c}
\Re e\left(\mathbf{A}_{1}\right) \\
\Im m\left(\mathbf{A}_{1}\right) \\
\vdots \\
\Re e\left(\mathbf{A}_{l}\right) \\
\Im m\left(\mathbf{A}_{l}\right)
\end{array}\right] .
$$

\section{Main Assumptions}

In this study, the following conditions are assumed to hold.

AS1) The $2 l n_{r} \times 2 n$ equivalent channel $\left(\mathbf{I}_{l} \otimes \overline{\mathbf{H}}\right) \mathbf{M}_{\mathcal{C}}$ is of full-column rank.

AS2) The noise vector $\mathbf{b}_{v}(u)$ is a complex, stationary, and ergodic Gaussian vector process, independent of the signals, with zero mean and covariance matrix given by $\sigma^{2} \mathbf{I}_{n_{r}}$, i.e., $\mathbf{b}_{v}(u) \sim \mathcal{N}_{c}\left(0, \sigma^{2} \mathbf{I}_{n_{r}}\right)$ where $\mathcal{N}_{c}(\mu, \Sigma)$ denotes a complex multivariate Gaussian distribution with mean $\mu$ and covariance $\Sigma$.

AS3) The transmitted symbols $s$ are independent and identically distributed (i.i.d.) and belong to a constellation $\mathcal{M}$ composed of $M$ states. The average symbol energy $E\left[|s|^{2}\right]$ is normalized ${ }^{2}$ to 1 and the real and imaginary parts of $s$ are assumed to be uncorrelated with variance $E\left[|s|^{2}\right] / 2=0.5$.

AS4) The receiver intercepts a whole number $N_{b}$ of space-time blocks $\mathbf{Y}=\left[\mathbf{Y}_{1}, \cdots, \mathbf{Y}_{N_{b}}\right]$, i.e., the first and last intercepted samples correspond to the start and the end of a space-time block, respectively.

In our study, the assumption AS1) is only required when the channel and noise power are unknown. As $\operatorname{rank}\left(\mathbf{M}_{\mathcal{C}}\right)=2 n$ for most of the well-designed STBCs, ${ }^{3}$ this assumption holds if $\operatorname{rank}(\mathbf{H})=n_{t}$. If $\operatorname{rank}(\mathbf{H})=n_{r}$, the inequality $n_{r} \geq(n / l)$ is a necessary condition to meet the requirement AS1). The condition AS2) is usual and AS3) holds for most of the complex constellations including $\geq 4$ phase-shift keying (PSK) and square

\footnotetext{
${ }^{2}$ Without loss of generality, the average symbol energy can be absorbed into the channel matrix $\overline{\mathbf{H}}$

${ }^{3}$ If $\mathbf{M}_{\mathcal{C}}$ fails to have full-column rank, the rank deficiency represents the number of wasted transmit diversity degrees. Such a code design is undesirable in practice [10].
} 
quadrature amplitude modulation (QAM). Finally, AS4) is assumed to allow simplifications of the following mathematical expressions. However, extensions of the proposed methods can be easily obtained when AS4) does not hold.

\section{STBC Recognition With Likelihood Ratio Tests}

The study deals with the recognition of the STBC in order to answer the following question: Given a measurement of $N$ received column vectors $\mathbf{Y}$, how can one find the STBC of the transmitted signals among a set $\Theta$ of STBC candidates? In the ML-based approach, the problem is viewed as a multiple-hypothesis problem. Under the assumption that the $a$ priori probabilities of the STBC candidates are equal, the recognized $\mathrm{STBC}, \widehat{\mathcal{C}}$ is the one that maximizes the log-likelihood function, i.e.,

$$
\widehat{\mathcal{C}}=\arg \max _{\mathcal{C} \in \Theta} \log (\Lambda[\mathbf{Y} \mid \mathcal{C}, \mathcal{X}])
$$

where $\log (\Lambda[\mathbf{Y} \mid \mathcal{C}, \mathcal{X}])$ is the $\log$-likelihood function of $\mathbf{Y}$ conditioned on the STBC $\mathcal{C}$ and on the communication parameters $\mathcal{X}$. Given that the conditions AS3) and AS4) hold, the log-likelihood function can be expressed as

$$
\log (\Lambda[\mathbf{Y} \mid \mathcal{C}, \mathcal{X}])=\sum_{v=1}^{N_{b}} \log \left(\Lambda\left[\tilde{\mathbf{y}}_{v} \mid \mathcal{C}, \mathcal{X}\right]\right)
$$

where $N_{b}=(N / l)$ is the total number of received blocks under the assumption $\mathcal{C}$ and $\log \left(\Lambda\left[\tilde{\mathbf{y}}_{v} \mid \mathcal{C}, \mathcal{X}\right]\right)$ is the log-likelihood function of the received block $\tilde{\mathbf{y}}_{v}$.

\section{The OPTIMAL ClassiFIER}

In this section, the optimal ML-based approach for modulation-classification [20], [21] is adapted to the STBC-recognition problem. If the channel matrix $\mathbf{H}$, the modulation $\mathcal{M}$, and the noise power $\sigma^{2}$ are perfectly known at the receiver side, then this method provides an upper bound on the performances of any classifier.

\section{A. Likelihood Function}

Let us model the unknown transmitted symbols $\mathbf{S}$ as random variables with a probability density function (pdf) equal to $f(\mathbf{s})$. The likelihood function $\Lambda\left[\tilde{\mathbf{y}}_{v} \mid \mathcal{C}, \mathbf{H}, \sigma^{2}, \mathcal{M}\right]$ is determined by averaging the conditional likelihood function $\Lambda\left[\tilde{\mathbf{y}}_{v} \mid \mathcal{C}, \mathbf{H}, \sigma^{2}, \mathbf{s}\right]$ with respect to the prior distribution of $\mathbf{s}$ so that

$$
\Lambda\left[\tilde{\mathbf{y}}_{v} \mid \mathcal{C}, \mathbf{H}, \sigma^{2}, \mathcal{M}\right]=\int_{\mathbf{s}} \Lambda\left[\tilde{\mathbf{y}}_{v} \mid \mathcal{C}, \mathbf{H}, \sigma^{2}, \mathbf{s}\right] f(\mathbf{s}) d \mathbf{s} .
$$

The $n$ elements of the vector $\mathbf{s}$ belong to a discrete alphabet $\mathcal{M}$ composed of $M$ states [assumption AS3)]. As these elements are i.i.d., one obtains $f(\mathbf{s})=1 / M^{n}$ for $\mathbf{s} \in \mathcal{M}^{n}$ and $f(\mathbf{s})=0$ elsewhere. The use of these equalities in (16) leads to

$$
\Lambda\left[\tilde{\mathbf{y}}_{v} \mid \mathcal{C}, \mathbf{H}, \sigma^{2}, \mathcal{M}\right]=\frac{1}{M^{n}} \sum_{\mathbf{s} \in \mathcal{M}^{n}} \Lambda\left[\tilde{\mathbf{y}}_{v} \mid \mathcal{C}, \mathbf{H}, \sigma^{2}, \mathbf{s}\right]
$$

where $\Lambda\left[\tilde{\mathbf{y}}_{v} \mid \mathcal{C}, \mathbf{H}, \sigma^{2}, \mathbf{s}\right]$ is the likelihood function of $\mathbf{Y}_{v}$ conditioned on $\mathcal{C}, \mathbf{H}, \sigma^{2}$, and $\mathbf{s}$. In the case of Gaussian distributed noise [assumption AS2)], one gets

$$
\begin{aligned}
& \log \left(\Lambda\left[\mathbf{Y} \mid \mathcal{C}, \mathbf{H}, \sigma^{2}, \mathcal{M}\right]\right)=-N_{b} \log \left(M^{n}\left(\pi \sigma^{2}\right)^{n_{r} l}\right) \\
& +\sum_{v=1}^{N_{b}} \log \left(\sum_{\mathbf{s} \in \mathcal{M}^{n}} \exp \left[-\frac{\left\|\tilde{\mathbf{y}}_{v}-\left(\mathbf{I}_{l} \otimes \overline{\mathbf{H}}\right) \mathbf{M}_{\mathcal{C}} \tilde{\mathbf{s}}\right\|_{F}^{2}}{\sigma^{2}}\right]\right)
\end{aligned}
$$

where $\|\cdot\|_{F}^{2}$ is the Frobenius norm. Finally, the STBC chosen $\widehat{\mathcal{C}}$ is the one that maximizes the function $\log \left(\Lambda\left[\mathbf{Y} \mid \mathcal{C}, \mathbf{H}, \sigma^{2}, \mathcal{M}\right]\right)$ over the set $\Theta$.

\section{B. Discussion}

One should note that, though this classifier maximizes the average probability of correct classification, it has several drawbacks. First, the log-likelihood function is computation time consuming. Let us denote by $O(1)$ the complexity of the elementary operations. It can be shown that the computation of the likelihood function has complexity $O\left(4 n_{t} N\left(n+n_{r} l\right) M^{n}\right)$. Therefore, the optimal classifier is computationally too complex in the case of high-order modulation $\mathcal{M}$ and/or a large number of symbols per block $n$ [21]. Furthermore, the computation of $\log \left(\Lambda\left[\mathbf{Y} \mid \mathcal{C}, \mathbf{H}, \sigma^{2}, \mathcal{M}\right]\right)$ requires the knowledge of several parameters, which are usually unknown in a noncooperative environment. When these parameters are unknown, this classifier is impractical since the maximization of the likelihood function with respect to $\mathcal{C}, \mathbf{H}, \sigma^{2}$, and $\mathcal{M}$ is computationally cost prohibitive.

\section{THE SOS-STBC CLASSIFIER}

The i.i.d. assumption AS3) and the signal model in (12) both show that the received vector $\tilde{\mathbf{y}}_{v}$ is the sum of $2 n$ i.i.d. random variables plus the additive noise. According to the central limit theorem, the distribution of $\tilde{\mathbf{y}}_{v}$ can be approximated by a Gaussian distribution for $2 n \rightarrow \infty$ whatever the modulation [13]. Although this approximation is only strictly correct in the asymptotic case, a recent study [29] showed that the Gaussian approximation provides the optimum second-order solution when: 1) the SNR is very low or 2) the symbols belong to a multilevel constellation. These considerations lead us to relax the finite alphabet constraint of the sources, which are modeled as i.i.d. Gaussian variables. One should note that this relaxation has been previously used with success in SOS-based channel estimation problems [11], [13]. In this study, this relaxation is employed to propose an SOS-STBC classifier.

\section{A. Likelihood Function}

As the additive noise $\tilde{\mathbf{b}}_{v}$ and the transmitted symbols $\tilde{\mathbf{s}}_{v}$ have zero mean, $E\left[\tilde{\mathbf{y}}_{v}\right]=0$. Furthermore as AS3) holds, one obtains

$$
E\left[\tilde{\mathbf{s}}_{v} \tilde{\mathbf{s}}_{v}^{T}\right]=\frac{1}{2} \mathbf{I}_{2 n}
$$

As $\mathbf{b}_{v}(u) \sim \mathcal{N}_{c}\left(0, \sigma^{2} \mathbf{I}_{n_{r}}\right)$ [assumption AS2)], it can be shown that

$$
E\left[\tilde{\mathbf{b}}_{v} \tilde{\mathbf{b}}_{v}^{T}\right]=\frac{\sigma^{2}}{2} \mathbf{I}_{2 n_{r} l} .
$$


From (12), (19), and (20), the covariance matrix $\Sigma_{\mathcal{C}, \mathbf{H}, \sigma^{2}}$ of the received samples can be expressed as

$$
\begin{aligned}
\Sigma_{\mathcal{C}, \mathbf{H}, \sigma^{2}} & =E\left[\tilde{\mathbf{y}}_{v} \tilde{\mathbf{y}}_{v}^{T}\right] \\
& =\left(\mathbf{I}_{l} \otimes \overline{\mathbf{H}}\right) \mathbf{M}_{\mathcal{C}} E\left[\tilde{\mathbf{s}}_{v} \tilde{\mathbf{s}}_{v}^{T}\right] \mathbf{M}_{\mathcal{C}}^{T}\left(\mathbf{I}_{l} \otimes \overline{\mathbf{H}}^{T}\right)+E\left[\tilde{\mathbf{b}}_{v} \tilde{\mathbf{b}}_{v}^{T}\right] \\
& =\frac{1}{2}\left(\mathbf{I}_{l} \otimes \overline{\mathbf{H}}\right) \mathbf{M}_{\mathcal{C}} \mathbf{M}_{\mathcal{C}}^{T}\left(\mathbf{I}_{l} \otimes \overline{\mathbf{H}}^{T}\right)+\frac{\sigma^{2}}{2} \mathbf{I}_{2 n_{r} l} .
\end{aligned}
$$

By using the Gaussian approximation, $\tilde{\mathbf{y}}_{v} \sim \mathcal{N}\left(0, \Sigma_{\mathcal{C}, \mathbf{H}, \sigma^{2}}\right)$ where $\mathcal{N}()$ corresponds to a multivariate Gaussian distribution. Therefore, the likelihood function of $\tilde{\mathbf{y}}_{v}$ conditioned on the covariance matrix $\Sigma_{\mathcal{C}, \mathbf{H}, \sigma^{2}}$ is expressed as

$\Lambda\left[\tilde{\mathbf{y}}_{v} \mid \Sigma_{\mathcal{C}, \mathbf{H}, \sigma^{2}}\right]=\frac{1}{(2 \pi)^{n_{r} l}\left|\Sigma_{\mathcal{C}, \mathbf{H}, \sigma^{2}}\right|^{\frac{1}{2}}} \exp \left[-\frac{1}{2} \tilde{\mathbf{y}}_{v}^{T} \Sigma_{\mathcal{C}, \mathbf{H}, \sigma^{2}}^{-1} \tilde{\mathbf{y}}_{v}\right]$

where $|$.$| corresponds to the matrix determinant. From (22) and$ (15), the likelihood function of $\mathbf{Y}$ is equal to

$$
\begin{aligned}
\log \left(\Lambda\left[\mathbf{Y} \mid \Sigma_{\mathcal{C}, \mathbf{H}, \sigma^{2}}\right]\right)= & \sum_{v=1}^{N_{b}} \log \left(\Lambda\left[\tilde{\mathbf{y}}_{v} \mid \Sigma_{\mathcal{C}, \mathbf{H}, \sigma^{2}}\right]\right) \\
= & -N_{b} \log \left((2 \pi)^{n_{r} l}\left|\Sigma_{\mathcal{C}, \mathbf{H}, \sigma^{2}}\right|^{\frac{1}{2}}\right) \\
& -\frac{1}{2} \sum_{v=1}^{N_{b}} \tilde{\mathbf{y}}_{v}^{T} \Sigma_{\mathcal{C}, \mathbf{H}, \sigma^{2}}^{-1} \tilde{\mathbf{y}}_{v} .
\end{aligned}
$$

Given that

$$
\tilde{\mathbf{y}}_{v}^{T} \Sigma_{\mathcal{C}, \mathbf{H}, \sigma^{2}}^{-1} \tilde{\mathbf{y}}_{v}=\operatorname{Tr}\left[\Sigma_{\mathcal{C}, \mathbf{H}, \sigma^{2}}^{-1} \tilde{\mathbf{y}}_{v} \tilde{\mathbf{y}}_{v}^{T}\right]
$$

where $\operatorname{Tr}[$.$] is the trace function, it follows that$

$$
\begin{aligned}
& \log \left(\Lambda\left[\mathbf{Y} \mid \Sigma_{\mathcal{C}, \mathbf{H}, \sigma^{2}}\right]\right)=-N_{b} l n_{r} \log (2 \pi) \\
& \quad-\frac{N_{b}}{2}\left(\log \left(\left|\Sigma_{\mathcal{C}, \mathbf{H}, \sigma^{2}}\right|\right)-\frac{N_{b}}{2} \operatorname{Tr}\left[\Sigma_{\mathcal{C}, \mathbf{H}, \sigma^{2}}^{-1} \mathbf{R}\right]\right)
\end{aligned}
$$

where $\mathbf{R}$ is the estimated covariance matrix

$$
\mathbf{R}=\frac{1}{N_{b}} \sum_{v=1}^{N_{b}} \tilde{\mathbf{y}}_{v} \tilde{\mathbf{y}}_{v}^{T} .
$$

Finally, the chosen STBC $\widehat{\mathcal{C}}$ is the one that maximizes the function $\log \left(\Lambda\left[\mathbf{Y} \mid \Sigma_{\mathcal{C}, \mathbf{H}, \sigma^{2}}\right]\right)$ over the set $\Theta$.

\section{B. Discussion}

One should note that the SOS-STBC classifier has several advantages over the optimal one: indeed, the knowledge of the modulation $\mathcal{M}$ being not a prerequisite, it is more easily implemented since the likelihood function solely depends on SOS. Furthermore, the SOS-STBC classifier can be extended to the blind context as discussed below.

In the blind context, the log-likelihood function in (25) cannot be assessed directly since the covariance matrix $\Sigma_{\mathcal{C}, \mathbf{H}, \sigma^{2}}$ is unknown at the receiver side. However, the log-likelihood function can be approximated by using the procedure proposed hereafter. First, $\mathcal{C}$ is assumed to be true and the unknown parameters $\sigma^{2}$ and $\mathbf{H}$ are estimated. Then, these estimates, denoted $\widehat{\sigma}^{2}$ and $\widehat{\mathbf{H}}$, are used to compute $\Sigma_{\mathcal{C}, \widehat{\mathbf{H}}, \widehat{\sigma}^{2}}$. Finally, the STBC chosen is the one that maximizes the function $\log \left(\Lambda\left[\mathbf{Y} \mid \Sigma_{\mathcal{C}, \widehat{\mathbf{H}}, \widehat{\sigma}^{2}}\right]\right)$. This procedure is sometimes called hybrid-likelihood ratio test (HLRT) [21]. To obtain the estimate of $\sigma^{2}$ and $\mathbf{H}$, several techniques can be employed. If $\mathcal{C}$ is an OSTBC, the Kullback-matching approach in [13] leads to the optimal ML joint estimate of the channel and noise variance. In the general STBC context, the joint estimation is a challenging problem and a suboptimal alternative relies on the separate estimation of $\sigma^{2}$ and $\mathbf{H}$. Let us introduce the following proposition.

Proposition 1: Let us denote the eigenvalues of $\Sigma_{\mathcal{C}, \mathbf{H}, \sigma^{2}}$ by $\lambda_{1} \geq \cdots \geq \lambda_{2 n_{r} l}$. The smallest $2 n_{r} l-2 n$ eigenvalues of $\Sigma_{\mathcal{C}, \mathbf{H}, \sigma^{2}}$ are all equal to $\sigma^{2} / 2$, i.e.,

$$
\lambda_{2 n+1}=\lambda_{2 n+2}=\cdots=\lambda_{2 n_{r} l}=\frac{\sigma^{2}}{2} .
$$

Proof: On condition that AS1), AS3), and AS4) hold, (21) shows that the rank of $\Sigma_{\mathcal{C}, \mathbf{H}, 0}$ is $2 n$ or, which is equivalent, that the $2\left(n_{r} l-n\right)$ smallest eigenvalues of $\Sigma_{\mathcal{C}, \mathbf{H}, 0}$ are equal to zero. It follows, therefore, that the smallest $2\left(n_{r} l-n\right)$ eigenvalues of $\Sigma_{\mathcal{C}, \mathbf{H}, \sigma^{2}}$ are all equal to $\sigma^{2} / 2$.

Using the above proposition, an estimate of the noise variance can be obtained as [30]

$$
\widehat{\sigma}^{2}=\frac{1}{n_{r} l-n} \sum_{k=2 n+1}^{2 n_{r} l} \rho_{k}
$$

where $\rho_{1} \geq \cdots \geq \rho_{2 n_{r} l}$ are the eigenvalues of the estimated covariance matrix $\mathbf{R}$. Concerning the estimation of $\widehat{\mathbf{H}}$, several techniques are available in [8]-[11], [13], and [14], however, the method employed must not introduce additional ambiguities to those associated to the blind channel estimation from SOS. This requirement guarantees a correct estimation of $\Sigma_{\mathcal{C}, \mathbf{H}, \sigma^{2}}$, even in the presence of channel indetermination. As pointed out in [31], the approaches [8]-[10] do not meet this requirement.

\section{THE CP CLASSIFIER}

In many STBC classification problems, the blind identification of the three code parameters $n_{t}, n$, and $l$ is sufficient to distinguish between several STBCs. For example, the Alamouti code and an OSTBC3 can be distinguished through detection of the number of transmitter antennas $n_{t}$. Furthermore, the spatial multiplexing and the Alamouti code can be differentiated by their code length $l$. Finally, two codes with the same code length and using the same number of antennas at the transmitter side can be identified through detection of the number of symbols $n$ per space-time block. The blind detection of the number of transmitter antennas is a well-known problem, which has been investigated in numerous papers and reviewed in [6]. This section focuses on the blind recognition of both code length $l$ and on the number of encoded symbols per block $n$. This CP classifier only exploits a small portion of the redundancy introduced by the STBC, but it is well suited for the blind scenario.

Let us consider an $\operatorname{STBC}\left(n_{t}, n, l\right)$ that transmits $n$ symbols during $l$ time slots. From Proposition 1, the covariance matrix $\Sigma_{n, l}=E\left[\tilde{\mathbf{y}}_{v} \tilde{\mathbf{y}}_{v}^{T}\right]$ can be modeled as

$$
\Sigma_{n, l}=\sum_{k=1}^{2 n}\left(\lambda_{k}-\frac{\sigma^{2}}{2}\right) \mathbf{v}_{k} \mathbf{v}_{k}^{T}+\frac{\sigma^{2}}{2} \mathbf{I}_{2 n_{r} l}
$$


where $\lambda_{1} \geq \cdots \geq \lambda_{2 n}$ and $\mathbf{v}_{1}, \cdots, \mathbf{v}_{2 n}$ are the eigenvalues and the eigenvectors, respectively, of $\Sigma_{n, l}$. The study by Wax [5], originally developed for the blind detection of the number of antennas, leads us to propose a classifier based on information criteria. The problem addressed by information criteria for model selection is as follows: given a set of observations $\mathbf{Y}$ and a family of parameterized likelihood functions $\log \left(\Lambda\left[\mathbf{Y} \mid \Sigma_{n, l}\right]\right)$, select the model that best fit the data. When there are several competing models, information criteria select the model that maximizes the function $\mathcal{L}(n, l)$ defined as [32]

$$
\mathcal{L}(n, l)=\log \left(\Lambda\left[\mathbf{Y} \mid \widehat{\Sigma}_{n, l}\right]\right)-\phi(z)
$$

where $\widehat{\Sigma}_{n, l}$ is the ML estimate of $\Sigma_{n, l}$ and where $\phi(z)$ is a penalty factor that depends on the number of independently adjusted parameters $z$ within the model.

\section{A. Expression of $\log \left(\Lambda\left[\mathbf{Y} \mid \widehat{\Sigma}_{n, l}\right]\right)$}

Following Anderson [30], the ML estimates of $\mathbf{v}_{k}, \lambda_{k}$, and $\sigma^{2}$ are given by

$$
\begin{aligned}
\widehat{\mathbf{v}}_{k} & =\mathbf{u}_{k}, \quad k=1, \cdots, 2 n \\
\hat{\lambda}_{k} & =\rho_{k}, \quad k=1, \cdots, 2 n \\
\widehat{\sigma}^{2} & =\frac{1}{n_{r} l-n} \sum_{k=2 n+1}^{2 n_{r} l} \rho_{k}
\end{aligned}
$$

where $\rho_{1} \geq \cdots \geq \rho_{2 n_{r} l}$ and $\mathbf{u}_{1}, \cdots, \mathbf{u}_{2 n_{r} l}$ are the eigenvalues and the eigenvectors, respectively, of the sample covariance matrix $\mathbf{R}$ defined in (26). It follows that

$$
\begin{aligned}
\operatorname{Tr}\left[\widehat{\Sigma}_{n, l}^{-1} \mathbf{R}\right] & =2 n_{r} l \\
\left|\widehat{\Sigma}_{n, l}\right| & =\left(\frac{1}{2\left(n_{r} l-n\right)} \sum_{k=2 n+1}^{2 n_{r} l} \rho_{k}\right)^{2\left(n_{r} l-n\right)} \prod_{k=1}^{2 n} \rho_{k} .
\end{aligned}
$$

Finally, by using these values in (25), one obtains

$$
\begin{aligned}
& \log \left(\Lambda\left[\mathbf{Y} \mid \widehat{\Sigma}_{n, l}\right]\right)=-\frac{N_{b}}{2} \sum_{k=1}^{2 n} \log \left(\rho_{k}\right)-N_{b}\left(n_{r} l-n\right) \\
& \times \log \left(\frac{1}{2\left(n_{r} l-n\right)} \sum_{k=2 n+1}^{2 n_{r} l} \rho_{k}\right)-N_{b} n_{r} l(\log (2 \pi)+1) .
\end{aligned}
$$

\section{B. Expression of $\mathcal{L}(n, l)$ With Information Criterion}

The number of free parameters $z$ in (30) is obtained by counting the number of degrees of freedom of the matrix $\widehat{\Sigma}_{n, l}$. The covariance matrix model expressed in (29) allows one to obtain: 1) $2 n$ free parameters for the eigenvalues $\lambda_{k}, 2$ ) one free parameter for the smallest eigenvalue $\sigma^{2} / 2$, and 3) $2 n_{r} l \times 2 n$ free parameters for the eigenvectors $\mathbf{u}_{k}(1 \leq k \leq 2 n)$. However, some of these parameters are not totally independent; indeed, the eigenvectors are constrained to have unit norm and to be mutually orthogonal [5]. Fig. 1 illustrates the number of constraints due to the normalization and orthogonalization. For the eigenvectors, the number of free parameters is equal to

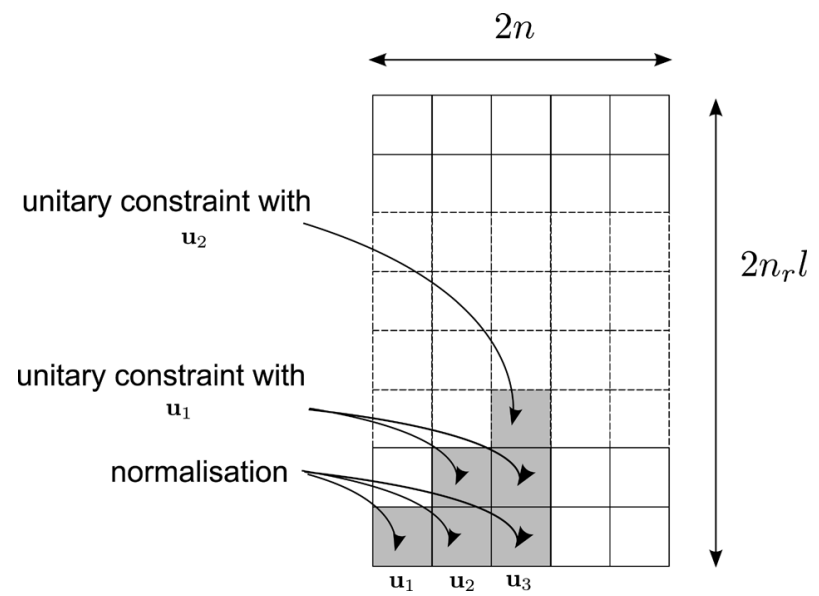

Fig. 1. Eigenvectors $\mathbf{u}_{k}$ : Number of free parameters.

$2 n_{r} l \times 2 n-(1+2+\cdots+2 n)=n\left(4 n_{r} l-2 n-1\right)$. Therefore, one obtains

$$
\begin{aligned}
z & =2 n+1+n\left(4 n_{r} l-2 n-1\right) \\
& =1+n\left(4 n_{r} l-2 n+1\right) .
\end{aligned}
$$

In (30), the form taken by the function $\phi(z)$ depends on the information criterion in use. In this study, the selected criterion is the Akaike information criterion (AIC), ${ }^{4}$ which is based on the concept of Kullback-Leiber divergence between two pdfs [34]. Its penalty function is equal to the number of free parameters, i.e., $\phi(z)=z$. In (37) and (36), leaving out terms that do not depend on the code parameters leads to

$$
\begin{aligned}
\mathcal{L}(n, l)= & -n\left(4 n_{r} l-2 n+1\right)-\frac{N_{b}}{2} \sum_{k=1}^{2 n} \log \left(\rho_{k}\right) \\
& -N_{b}\left(n_{r} l-n\right) \log \left(\frac{1}{2\left(n_{r} l-n\right)} \sum_{k=2 n+1}^{2 n_{r} l} \rho_{k}\right)
\end{aligned}
$$

where $l$ and $n$ are the block length and the number of encoded symbols per block, respectively, of the STBC $\mathcal{C}$. Finally, the code parameters $n$ and $l$ chosen are the ones that maximize the function $\mathcal{L}(n, l)$. One should note that, even if the $\mathrm{CP}$ classifier is designed for complex constellations [assumption AS3)], it can be extended to the context of real constellations by replacing $2 n$ with $n$ in (38).

\section{Discussion}

Compared to the SOS classifier, the advantage of the CP classifier is that no channel estimation is required in the decision problem, and the only key-parameters are the eigenvalues $\rho_{1} \geq$ $\ldots, \rho_{2 n_{r} l}$ of the estimated covariance matrix R. However, this classifier only exploits a small portion of the redundancy introduced by the STBC. In particular, the method is not able to discriminate between two STBCs that transmit the same number of

${ }^{4}$ For low and medium SNR, simulations have shown that the best average probability of correct detection is obtained with the AIC criterion. If the STBCs to be classified have the same block length, then the $\mathrm{CP}$ classifier is equivalent to the method [5] and a consistent criterion, like the minimum description length (MDL) [33] should be preferred. 
TABLE I

COST FunCTION AND PRIOR INFORMATION FOR EACH ClASSIFIER. SYMBOL $\oslash$ INDICATES THE EMPTY SET

\begin{tabular}{|c|c|c|}
\hline Classifier & Algorithm & $\begin{array}{c}\text { Prior } \\
\text { Information }\end{array}$ \\
\hline Optimal Classifier & Cost Function (18) & $\mathbf{H}, \sigma^{2}, \mathcal{M}$ \\
SOS-STBC & Cost Function (25) & $\mathbf{H}, \sigma^{2}$ \\
(blind) SOS-STBC & Cost Function (25) & $\oslash$ \\
(blind) CP Classifier & Cost Function (38) & $\oslash$ \\
(blind) Classifier [17] & See Reference [17] & $\oslash$ \\
(blind) Classifier [18] & See Reference [18] & $\oslash$ \\
\hline
\end{tabular}

symbols during the same time slots. This limitation is caused by the estimation of $\mathbf{u}_{k}(k=1, \cdots, 2 n)$; indeed, (31) shows that the eigenvector estimate does not take into account the specific structure of the STBC.

\section{Simulation RESULts}

To highlight the interest of this study, let us consider two STBC classification problems. The first is the recognition of $q=2$ STBCs that both use two antennas: the spatial multiplexing and the Alamouti code. Then, the second problem is about the recognition of $q=7$ STBCs with a higher number of transmit antennas $\left(n_{t}=4\right)$. To assess and compare the performances by the proposed algorithms, 1000 of Monte Carlo simulations were run for each STBC. The algorithms were implemented on Matlab, and the simulations were run on a Pentium dual core $\mathrm{PC}$ at $1.6 \mathrm{GHz}(\mathrm{RAM}=1 \mathrm{Go})$. Each simulation trial was carried out under the following conditions: 1) a Rayleigh distributed channel, i.e., each element of $\mathbf{H}$ follows an i.i.d. circular Gaussian distribution with zero mean and unit variance, 2) a QPSK modulation $(M=4)$, and 3$)$ a temporally and spatially zero-mean white Gaussian additive noise with $\sigma^{2}$ as variance.

The average probability of correct recognition $p_{c}$ was used to evaluate the performance of each classifier. When the $a$ priori probabilities of the STBC candidates are equal, this probability is defined as $p_{c}=(1 / q) \sum_{i=1}^{q} p\left(\Omega_{i} / \Omega_{i}\right)$ where $q$ is the number of STBC candidates and $p\left(\Omega_{i} / \Omega_{i}\right)$ is the conditional probability of choosing the STBC $\Omega_{i}$ when the STBC $\Omega_{i}$ was originally transmitted. The performances of our algorithms were assessed for several signal-to-noise ratios (SNRs). The total power of transmitted signals was constrained to $P=(1 / l) E\left[\operatorname{Tr}\left[\mathbf{C}(s) \mathbf{C}^{H}(s)\right]\right]$ regardless of the number of transmitter antennas $n_{t}$ and the SNR was defined as [3], [4]

$$
\mathrm{SNR}=10 \log _{10}\left(\frac{P}{\sigma^{2}}\right)
$$

\section{A. Recognition of $q=2$ STBCs Using $n_{t}=2$ Antennas}

The performances of the six classifiers reported in Table I were assessed for the recognition of the spatial multiplexing and Alamouti coding. Regarding the blind SOS-STBC classifier, the channel was estimated by using either a principal component analysis (PCA) or the algorithm stated in [11] according to the STBC assumption. One can notice that these two methods do not introduce additional ambiguities to those associated to the blind channel estimation from SOS [31]. The proposed ML-based classifiers are compared with the

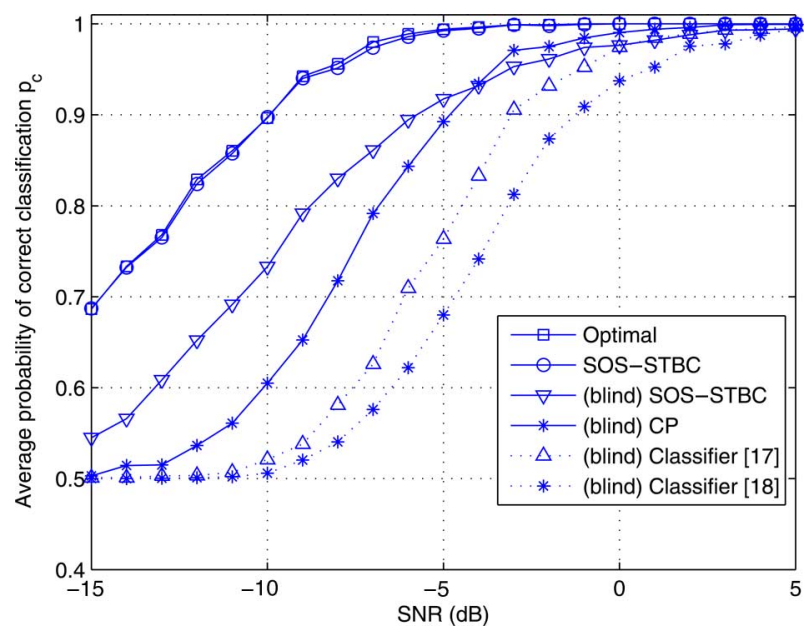

Fig. 2. Average probability of correct recognition $p_{c}$ for the classification of two STBCs using $n_{t}=2$ antennas. The receiver is composed of $n_{r}=3$ antennas and intercepts $N=512$ samples.

two classifiers described in [17] and [18]. These feature-based methods compute the norm of a space-time correlation matrix to characterize the space-time redundancy. Then, method [17] employs a statistical hypothesis test ${ }^{5}$ and a decision tree to perform the classification whereas method [18] selects the STBC which minimizes a cost function.

In the first simulation, the number of receiver antennas and the number of received samples are equal to $n_{r}=3>n_{t}$ and $N=512$, respectively. Fig. 2 presents the average probability of correct recognition for the six classifiers. It shows that the performances of the SOS-STBC classifier with perfect knowledge of $\mathbf{H}$ and $\sigma^{2}$ are nearly optimal and that the recognition is perfect at $\mathrm{SNR}=0 \mathrm{~dB}$. Regarding the blind methods, the best performances are obtained with the blind SOS-STBC classifier in low $\mathrm{SNR}$ regions. From $\mathrm{SNR}=-3 \mathrm{~dB}$, the $\mathrm{CP}$ classifier outperforms the blind SOS-STBC classifier. One can notice that the proposed blind SOS-STBC and CP classifiers both outperform those described in [17] and [18].

Fig. 3 displays the average probability of correct recognition for a receiver composed of $n_{r}=4$ antennas. Compared to Fig. 2, it shows that increasing the number of receiver antennas improves the probability of correct detection. To detail how the classifiers make error, Figs. 4 and 5 deals with the probability of correct identifications for the spatial multiplexing and the Alamouti code, respectively (i.e., the diagonal elements of the $2 \times 2$ confusion matrix). Concerning the optimal and SOS-STBC classifiers, the two codes are equally detected. The other algorithms, except [18], seem to favor the spatial multiplexing over the Alamouti code.

Fig. 6 presents the performances of each classifier for a small number of received samples $(N=64)$ and $n_{r}=4$. The performances of each classifier decrease compared to Fig. 3 $(N=512)$. Furthermore, one can notice that decreasing the number of samples increases the difference between the optimal and SOS-STBC classifiers.

From a practical point of view, it is important to access the complexity of each classifier. Unfortunately, it is difficult to ob-

\footnotetext{
${ }^{5}$ In our simulation, the probability of false alarm was fixed to $10^{-3}$
} 


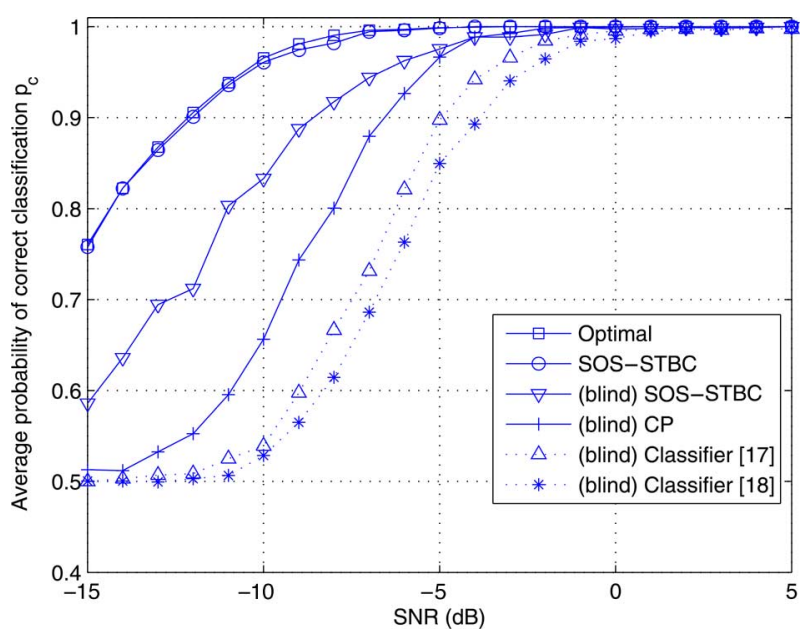

Fig. 3. Average probability of correct recognition $p_{c}$ for the classification of two STBCs using $n_{t}=2$ antennas. The receiver is composed of $n_{r}=4$ antennas and intercepts $N=512$ samples.

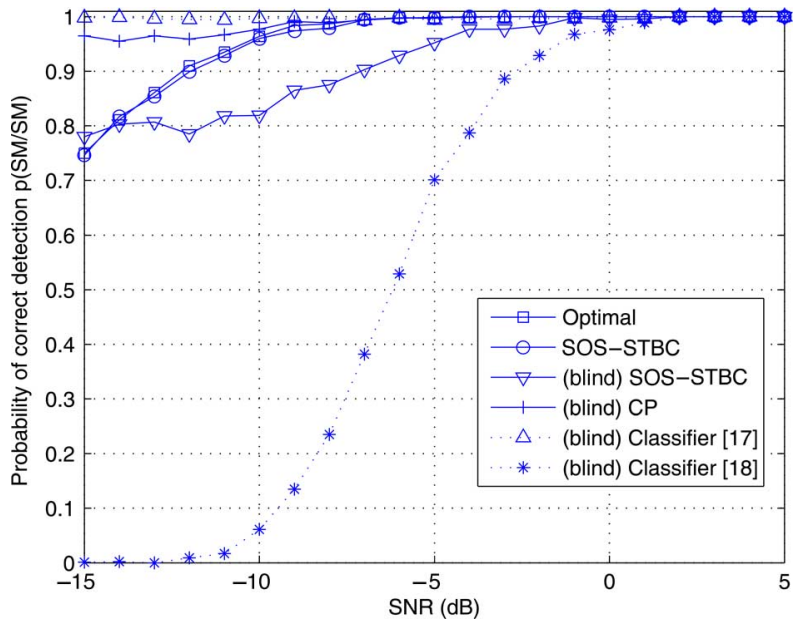

Fig. 4. Probability of correct detection for the spatial multiplexing. The receiver is composed of $n_{r}=4$ antennas and intercepts $N=512$ samples.

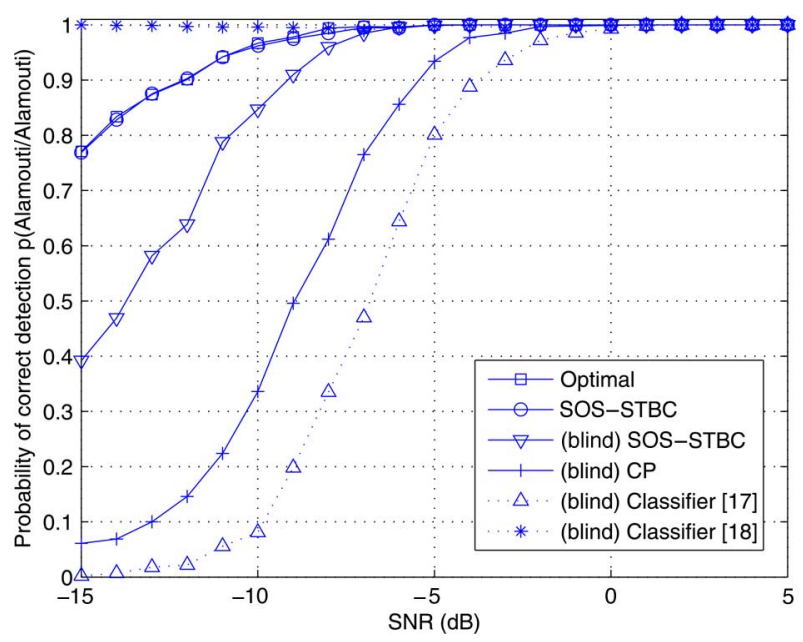

Fig. 5. Probability of correct detection for the Alamouti code. The receiver is composed of $n_{r}=4$ antennas and intercepts $N=512$ samples.

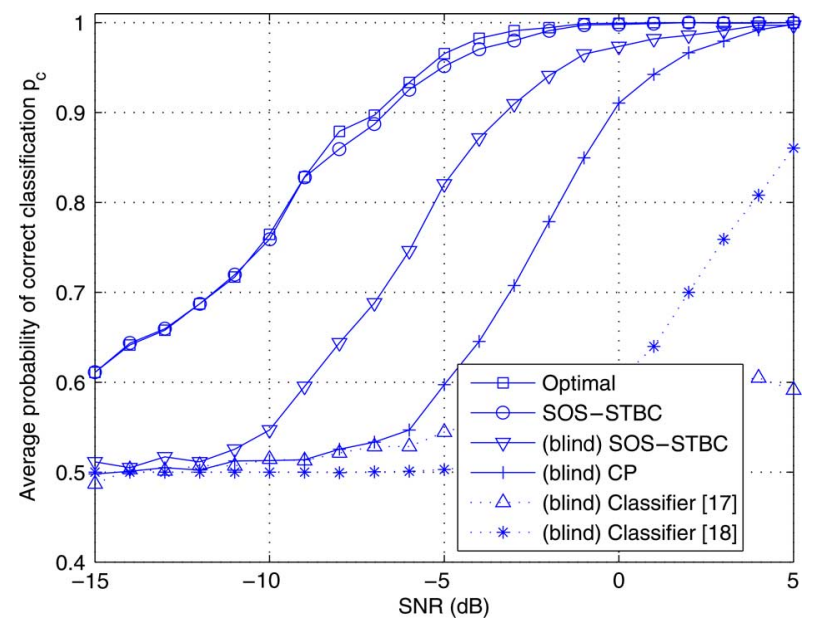

Fig. 6. Average probability of correct recognition $p_{c}$ for the classification of two STBCs using $n_{t}=2$ antennas. The receiver is composed of $n_{r}=4$ antennas and intercepts $N=64$ samples.

TABLE II

Average Computation Time for Each Monte Carlo Trial. ReCognition of Spatial Multiplexing and the Alamouti Code With a Receiver COMPOSED OF $n_{r}=4$ ANTENNAS $(N=512)$.

\begin{tabular}{|c|c|}
\hline Classifier & Computation time \\
\hline Optimal Classifier & $251.1 \times 10^{-3} \mathrm{~s}$ \\
SOS-STBC & $11.6 \times 10^{-3} \mathrm{~s}$ \\
(blind) SOS-STBC & $29.9 \times 10^{-3} \mathrm{~s}$ \\
(blind) CP Classifier & $3.0 \times 10^{-3} \mathrm{~s}$ \\
(blind) Classifier [17] & $15.8 \times 10^{-3} \mathrm{~s}$ \\
(blind) Classifier [18] & $9.1 \times 10^{-3} \mathrm{~s}$ \\
\hline
\end{tabular}

tain the exact number of elementary operations for the six classifiers. Most of them perform matrix operations (eigenvalue decomposition, inversion), which are usually computed through iterative methods. To determine the complexity, Table II presents the average computation time, in seconds, for each Monte Carlo trial. It shows that the optimal classifier is the most computation time consuming since $251 \mathrm{~ms}$ are needed to perform the classification. The SOS classifier is 21.5 times faster for the same level of performances; this speed difference is even more marked in the case of high-order modulation or of a larger number of symbols per block. In a blind context, the channel estimation step elevates the time required for computation by the SOS-STBC classifier. Finally, the CP proves to be the fastest and appears thus as well suited for real-time implementations. In particular, this algorithm is five times faster than the one presented in [17].

\section{B. Recognition of $q=7$ STBCs Using $n_{t}=4$ Antennas}

Let us consider the problem posed by the blind recognition of seven STBCs included in the IEEE 802.16e standard [35]:

- a 1/2-rate orthogonal code: $\operatorname{OSTBC}(4,4,8)$;

- a 3/4-rate orthogonal code: $\operatorname{OSTBC}(4,3,4)$;

- a 1-rate code: $\operatorname{STBC}(4,4,4)$;

- two 2-rate codes: $\operatorname{STBC}(4,8,4)$ and $\operatorname{STBC}(4,4,2)$;

- a 3-rate code: $\operatorname{STBC}(4,6,2)$;

- a 4-rate code: $\operatorname{STBC}(4,4,1)$ (spatial multiplexing).

For this classification problem, the use of the optimal classifier is totally impractical because of its computational cost. Regarding 


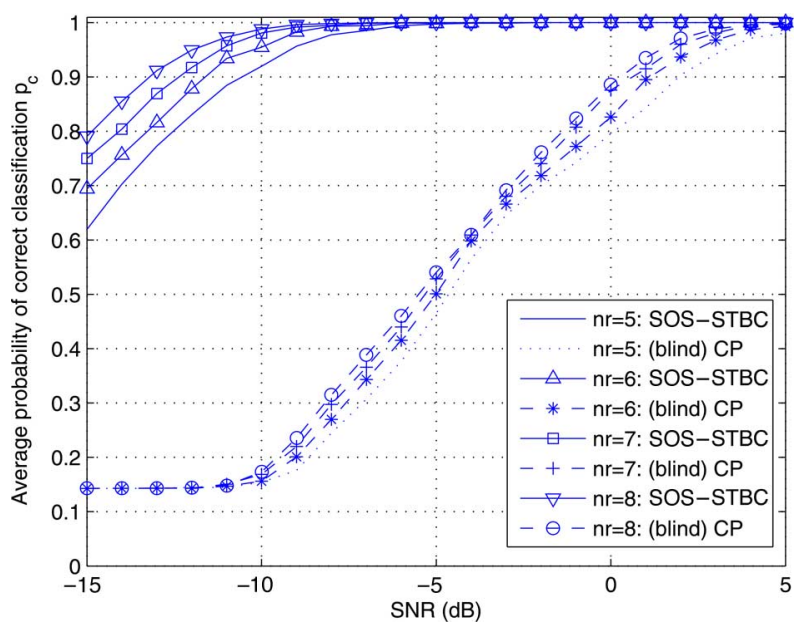

Fig. 7. Average probability of correct recognition $p_{c}$ for the classification of seven STBCs using $n_{t}=4$ antennas. The receiver intercepts $N=512$ samples and $n_{r}=\{5,6,7,8\}$.

the SOS-STBC classifier, its application requires the channel knowledge. In the blind context, several methods are available in literature for channel estimation [8]-[10], [14], however, as pointed out in [31], the blind channel algorithms [8]-[10] introduce additional ambiguities to those associated to the SOSbased channel estimation problem. Furthermore, it can be shown that method [14] does not respect condition (19) for nonorthogonal and nonidentifiable STBC. Therefore, the matrix $\Sigma_{\mathcal{C}, \mathbf{H}, \sigma^{2}}$ cannot be estimated with the methods [8]-[10], and [14] and the blind SOS-STBC classifier is impractical. Finally, the two classifiers described in [18] and [17] are unable to distinguish between the $\operatorname{STBC}(4,6,2)$ and the $\operatorname{STBC}(4,4,2)$ because the space-time correlation profiles displayed per both codes are alike. These considerations thus lead us to, hereafter, only report on the performances by the SOS-STBC and blind CP classifiers.

Fig. 7 depicts the average probability of correct recognition versus the number of receiver antennas $\left(n_{r}=\{5,6,7,8\}\right)$. As previously discussed, increasing $n_{r}$ improves the classification performances. With $n_{r}=8$, the figure shows that the seven STBCs are perfectly identified by the SOS-STBC classifier at $\mathrm{SNR}=-5 \mathrm{~dB}$. Regarding the blind CP classifier, the average probability of correct detection tends to 0.9995 with $n_{r}=8$ at large SNR. More precisely, the probability of choosing the $\operatorname{STBC}(4,4,4)$ when the $\operatorname{OSTBC}(4,3,4)$ was originally transmitted and the probability of choosing the $\operatorname{STBC}(4,6,2)$ when the $\operatorname{STBC}(4,4,2)$ was originally transmitted are close but not equal to 0 even for high SNR. These errors are caused by the asymptotic properties of the Akaike criterion [5], which tends to overestimate the number of symbols per block, $2 n$, if the STBC candidates have the same block length $l$. If the STBCs to be distinguished have the same parameter $l$, better asymptotic performances could be obtained with a consistent criterion like the MDL [5].

Figs. 8 and 9 present the probability of correct detection of each code with $n_{r}=8$ receiver antennas. Concerning the performances obtained with the SOS-STBC classifier, Fig. 8 shows that the $\operatorname{OSTBC}(4,4,8)$ is the first STBC to be detected. It is followed by the $\operatorname{STBC}(4,4,4)$ and then the $\operatorname{OSTBC}(4,3,4)$, the $\operatorname{STBC}(4,4,2)$, the $\operatorname{STBC}(4,4,1)$, and the $\operatorname{STBC}(4,6,2)$.

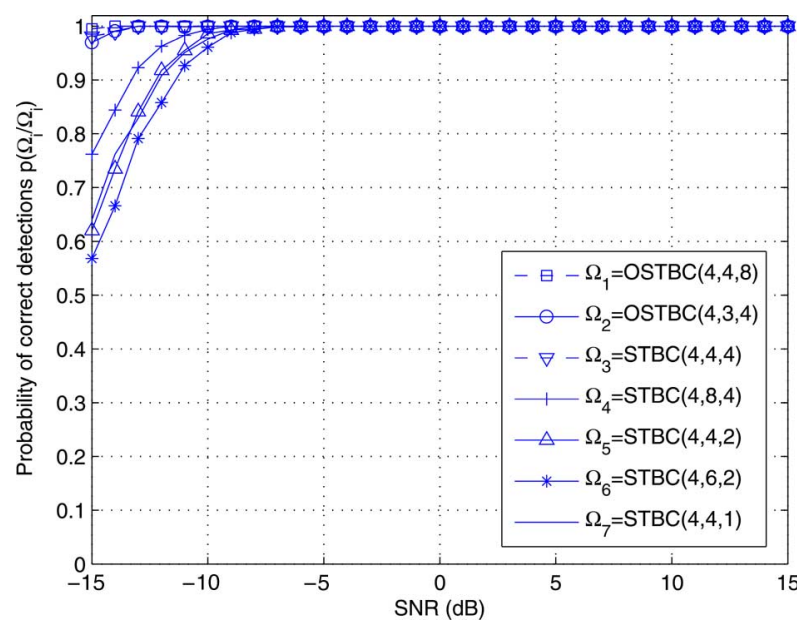

Fig. 8. SOS-STBC classifier: probability of correct detection $p\left(\Omega_{i} / \Omega_{i}\right)$. The receiver is composed of $n_{r}=8$ antennas and intercepts $N=512$ samples.

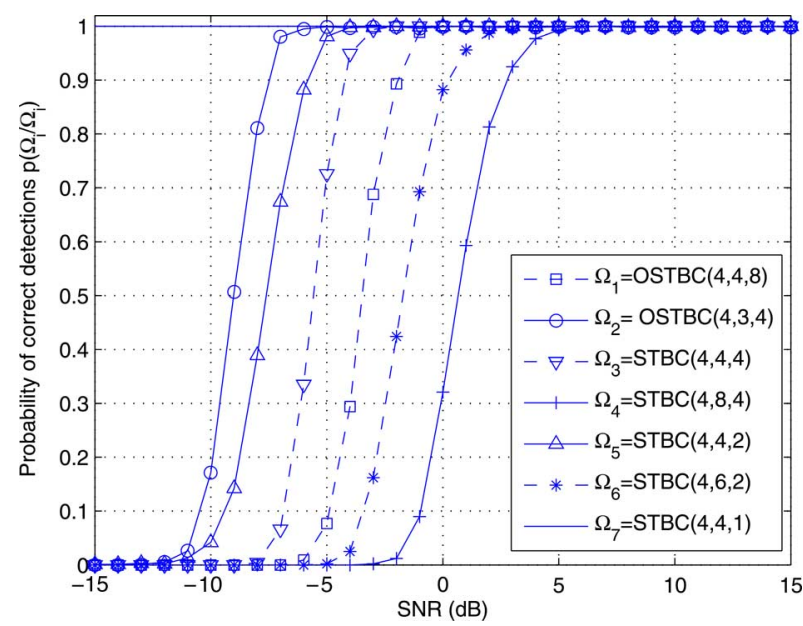

Fig. 9. Blind CP classifier: probability of correct detection $p\left(\Omega_{i} / \Omega_{i}\right)$. The receiver is composed of $n_{r}=8$ antennas and intercepts $N=512$ samples.

TABLE III

CP Classifier: RANKING ORdER AND PENALTy FunCtion. THE RECEIVER IS COMPOSED OF $n_{r}=8$ ANTENNAS

\begin{tabular}{|c||c||c|c||c|}
\hline $\begin{array}{c}\text { Ranking } \\
\text { order }\end{array}$ & STBC & $n$ & $l$ & $\begin{array}{c}\phi(z)=z \\
\left(n_{r}=8\right)\end{array}$ \\
\hline 1 & STBC $(4,4,1)$ & 4 & 1 & 101 \\
2 & OSTBC $(4,3,4)$ & 3 & 4 & 370 \\
3 & STBC $(4,4,2)$ & 4 & 2 & 229 \\
4 & STBC $(4,4,4)$ & 4 & 4 & 485 \\
5 & OSTBC $(4,4,8)$ & 4 & 8 & 997 \\
6 & STBC $(4,6,2)$ & 6 & 2 & 319 \\
7 & STBC $(4,8,4)$ & 8 & 4 & 905 \\
\hline
\end{tabular}

Regarding the blind $\mathrm{CP}$ classifier, it recognizes only the spatial multiplexing at $\mathrm{SNR}=-15 \mathrm{~dB}$. Then, the algorithm detects the $\operatorname{OSTBC}(4,3,4)$, the $\operatorname{STBC}(4,4,2)$, the $\operatorname{STBC}(4,4,4)$, the $\operatorname{OSTBC}(4,4,8)$, the $\operatorname{STBC}(4,6,2)$, and the $\operatorname{STBC}(4,8,4)$. Table III presents the ranking order and the penalty function for each code. It shows that, for two codes with different $l$ and similar $n$, the CP classifier seems to favor the STBC with the lowest penalty function $\phi(z)$. As a larger value of the block length $l$ increases the penalty function [see (37)], it indicates a preference 
for the STBC with the lowest block length. Similarly, for two codes with a different number of symbols per block and with the same code length, the CP classifier seems to favor the STBC with the lowest penalty function $\phi(z)$, i.e., with the smallest $n$.

\section{CONCLUSION}

The investigations reported in this paper concerned the recognition of STBCs by likelihood-based classifiers. Three newly developed classifiers referred to as optimal, SOS-STBC, and CP classifiers were presented. Though the first classifier is optimal in an ideal context, it is impractical when the communication parameters are unknown at the receiver side. In the blind scenario, the SOS-STBC proved to outperform the CP classifier in low SNR regions, but its application requires the channel to be estimated. On the other hand, the knowledge of this parameter is not a prerequisite for the $\mathrm{CP}$ classifier, which can be employed to distinguish between several STBCs with different CPs. The simulations carried out in this study showed that the SOS-STBC and $\mathrm{CP}$ classifiers lead to a better average probability of correct recognition than the classifiers described in literature. Future investigations will focus on the theoretical analysis of the classifier performances.

\section{ACKNOWLEDGMENT}

The authors would like to thank M.-P. Friocourt and J. Leschaeve for their help in checking the English language usage, as well as the associate editor and the anonymous reviewers for their useful comments.

\section{REFERENCES}

[1] C. Oestges and B. Clerckx, MIMO Wireless Communications: From Real Word Propagation to Space-Time Code Design. New York: Academic, 2007.

[2] E. Larsson and P. Stoica, Space-Time Block Coding for Wireless Communication. Cambridge, U.K.: Cambridge Univ. Press, 2003.

[3] B. Vucetic and J. Yuan, Space-Time Coding. New York: Wiley, 2003.

[4] L. Tran, T. Wysocky, A. Mertins, and J. Seberry, Complex Orthogonal Space-Time Processing in Wireless Communications. New York: Springer-Verlag, 2006.

[5] M. Wax and T. Kailath, "Detection of signals by information theoretic criteria," IEEE Trans. Acoust. Speech Signal Process., vol. 33, no. 2, pp. 387-392, 1985.

[6] S. Aouada, A. Zoubir, and S. Cee, "A comparative study on source number detection," in Proc. IEEE Inf. Sci. Signal Process. Appl., Paris, France, 2003, vol. 1, pp. 173-176.

[7] A. Swami, S. Barbarossa, and B. Sadler, "Blind source separation and signal classification," in Proc. Asilomar Conf. Signals Syst. Comput., Pacific Grove, CA, 2000, vol. 2, pp. 1181-1191.

[8] A. Swindlehurst and G. Leus, "Blind and semi-blind equalization for generalized space-time block codes," IEEE Trans. Signal Process., vol. 50, no. 10, pp. 2489-2498, Oct. 2002.

[9] E. Larsson, P. Stoica, and J. Li, "Orthogonal space-time block codes: Maximum likelihood detection for unknown channels and unstructured intereferences," IEEE Trans. Signal Process., vol. 51, no. 2, pp. 362-372, Feb. 2003.

[10] N. Ammar and Z. Ding, "Blind channel identifiability for generic linear space-time block codes," IEEE Trans. Signal Process., vol. 55, no. 1, pp. 202-217, Jan. 2007.

[11] S. Shahbazpanahi, A. Gershman, and J. Manton, "Closed form blind MIMO channel estimation for othogonal space-time codes," IEEE Trans. Signal Process., vol. 53, no. 12, pp. 4506-4517, Dec. 2005.

[12] W. Ma, B. Vo, T. Davidson, and P. Ching, "Blind ML detection of orthogonal space-time block codes: Efficient high-performance implementations," IEEE Trans. Signal Process., vol. 54, no. 2, pp. 738-751, Feb. 2006.
[13] J. Via and I. Santamaria, "Correlation matching approaches for blind OSTBC channel estimation," IEEE Trans. Signal Process., vol. 56, no. 12, pp. 5950-5961, Dec. 2008.

[14] J. Via, I. Santamaria, and J. Perez, "Code combination for blind channel estimation in general MIMO-STBC systems," EURASIP J. Advances Signal Process., vol. 2009, no. 3, 2009.

[15] M. Shi, Y. Bar-Ness, and W. Su, "STC and BLAST MIMO modulation recognition," in Proc. IEEE GLOBECOM Conf., Washington, DC, 2007, pp. 3034-3039.

[16] M. D. Young, R. Health, and B. L. Evans, "Using higher order cyclostationarity to identify space-time block codes," in Proc. IEEE GLOBECOM Conf., New Orleans, LA, 2008, pp. 3370-3374.

[17] V. Choqueuse, K. Yao, L. Collin, and G. Burel, "Hierarchical space time block code recognition using correlation matrices," IEEE Trans. Wireless Commun., vol. 7, no. 9, pp. 3526-3534, Sep. 2008.

[18] V. Choqueuse, K. Yao, L. Collin, and G. Burel, "Blind recognition of linear space time block codes," in Proc. IEEE Int. Conf. Acoust. Speech Signal Process., Las Vegas, NV, 2008, pp. 2833-2836.

[19] A. Polydros and K. Kim, "On the detection and classification of quadrature digital modulations in broad-band noise," IEEE Trans. Commun., vol. 38, no. 8, pp. 1199-1211, Aug. 1990.

[20] W. Wei and M. Mendel, "Maximum likelihood classification for digital amplitude-phase modulations," IEEE Trans. Commun., vol. 48, no. 2, pp. 189-193, Feb. 2000.

[21] O. Dobre, Y. Bar-Ness, and W. Su, "Blind modulation classification: A concept whose time has come," in Proc. IEEE Sarnoff Symp. Advances Wired Wireless Commun., Princeton, NJ, 2005, pp. 223-228.

[22] B. Hassibi and B. Hochwald, "High-rate codes that are linear in space and time," IEEE Trans. Inf. Theory, vol. 48, no. 7, pp. 1804-1824, Jul. 2002.

[23] H. Jafarkhani, "A quasi-orthogonal space-time block code," IEEE Trans. Commun., vol. 49, no. 1, pp. 1-4, Jan. 2001.

[24] S. Alamouti, "A simple transmit diversity technique for wireless communication," IEEE J. Sel. Areas Commun., vol. 16, no. 8, pp. 1451-1458, Aug. 1998.

[25] V. Tarokh, H. Jafarkhani, and A. Calderbank, "Space-time block codes from orthogonal designs," IEEE Trans. Inf. Theory, vol. 45, no. 5, pp. 744-765, Sep. 1999.

[26] Y. Wu and S. Chan, "On the symbol timing recovery in space-time coding systems," in Proc. IEEE Wireless Commun. Netw. Conf., New Orleans, LA, 2003, pp. 420-424.

[27] P. Ciblat, P. Loubaton, E. Serpedin, and G. Giannakis, "Asymptotic analysis of blind cyclic correlation-based symbol-rate estimator," IEEE Trans. Inf. Theory, vol. 48, no. 7, pp. 1922-1934, Jul. 2002.

[28] J. Brewer, "Kronecker products and matrix calculus in system theory," IEEE Trans. Circuits Syst., vol. CAS-25, no. 9, pp. 772-781, Sep. 1978.

[29] J. Villares and G. Vazquez, "The Gaussian assumption in second-order estimation problems in digital communications," IEEE Trans. Signal Process., vol. 55, no. 10, pp. 4994-5002, Oct. 2007.

[30] T. W. Anderson, "Asymptotic theory for principal component analysis," Ann. J. Math. Statist., vol. 34, pp. 122-148, 1963.

[31] J. Via and I. Santamaria, "On the blind identifiablity of orthogonal space time block codes from second order statistics," IEEE Trans. Inf. Theory, vol. 54, no. 2, pp. 709-722, Feb. 2008.

[32] S. Konishi and G. Kitagawa, Information Criteria and Statistical Modeling. New York: Springer-Verlag, 2008.

[33] J. Rissanen, "Modeling by shortest data description length," Automatica, vol. 14, pp. 461-471, 1978.

[34] H. Akaike, "A new look at the statistical model identification," IEEE Trans. Autom. Control, vol. AC-19, no. 6, pp. 716-723, Jun. 1974.

[35] Standard for Local and Metropolitan Area Networks, Part 16: Air Interface for Fixed and Mobile Broadband Wireless Access System, IEEE $802.16 \mathrm{e}, 2005$

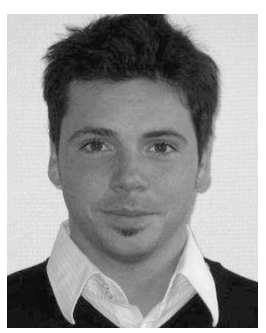

Vincent Choqueuse (M'08) was born in 1981 in Brest, France. He received the Dipl.-Ing. degree in computer science and the M.Sc. degree in statistics from Troyes University of Technology (UTT), France, in 2004 and 2005, respectively, and the Ph.D. degree in digital communications from University of Brest, France, in 2008.

Since September 2009, he has been an Associate Professor at the University Technology Institute (IUT), Brest, France, and a member of the Laboratory LBMS (EA 4325). His current research interests focus on signal processing and statistics for communications, diagnosis, and digital audio. 


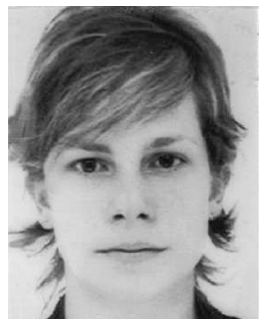

Mélanie Marazin was born in 1983. She received the M.Sc. degree in sciences and technologies of telecommunications from University of Brest, Brest, France, in 2006, where she is currently working towards the Ph.D. degree in digital communications.

She is a member of the Laboratory for Sciences and Technologies of Information, Communication and Knowledge (Lab-STICC-UMR CNRS 3192), Brest, France, and her research interests focus on communications intelligence (COMINT) and blind recognition of codes. She also teaches digital communications, networks, electronics, and computer sciences at the University of Brest.

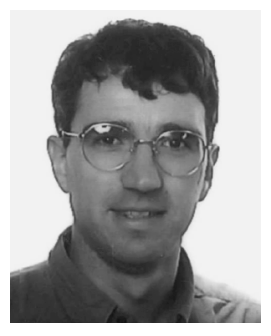

Ludovic Collin received the Ph.D. degree in electrical engineering from the University of Bretagne Occidentale, Brest, France, in 2002.

From 1989 to 1999, he was with ORCA Instrumentation, Brest, France, where he developed oceanographical instrumentation and acoustic modems. From 1999 to 2002, he was Research and Teaching Assistant at French Naval Academy, Lanveoc, France, and at the Institute of Technology of Lannion. From 2003 to 2007, he was Assistant Professor at the ENSIETA, Brest, France. Since 2007, he has been Assistant Professor at the University of Brest, Brest, France, and member of the Laboratory for Science and Technologies of Information, Communication and Knowledge (Lab-STICC-UMR CNRS 3192). His research interests are in MIMO systems and interception of communications.

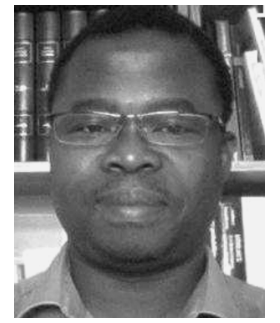

Koffi Clément Yao received the Ph.D. degree in optical signal processing and computer sciences from University Louis Pasteur of Strasbourg, France, in 1990.

After his postdoctorate research on optical neural networks at Ecole Nationale Supérieure des Télécommunications of Brest, he joined the French Naval Academy as Assistant Professor in Statistical Signal Processing in 1992. His research interest was focused on pattern recognition and blind signal separation in underwater acoustics. Since 2001, he has been Assistant Professor at the University of Brest, Brest, France. His present research interests are in MIMO systems and blind interception of digital communication signals.

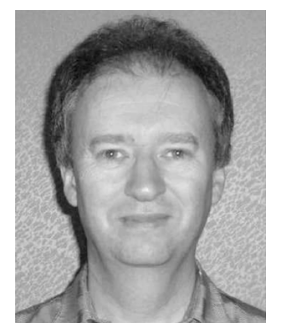

Gilles Burel (M'00-SM'08) was born in 1964. He received the M.Sc. degree from Ecole Supérieure d'Electricité, Gif Sur Yvette, France, in 1988, the Ph.D. degree from University of Brest, Brest, France, 1991, and the Habilitation to Supervise Research degree in 1996.

From 1988 to 1997, he was a member of the technical staff of Thomson CSF, then Thomson Multimedia, Rennes, France, where he worked on image processing and pattern recognition applications as project manager. Since 1997, he has been Professor of Digital Communications, Image and Signal Processing at the University of Brest. He is Associate Director of the Laboratory for Science and Technologies of Information, Communication and Knowledge (Lab-STICC-UMR CNRS 3192). He is author or coauthor of 19 patents, one book, and 140 scientific papers. His current research interests are in signal processing for digital communications, MIMO systems, and interception of communications. 